\title{
Kernos
}

Revue internationale et pluridisciplinaire de religion grecque antique

6 | 1993

Varia

\section{A. Avagianou, Sacred Marriage in the Rituals of Greek Religion}

Vinciane Pirenne-Delforge

\section{(2) OpenEdition \\ Journals}

Édition électronique

URL : http://journals.openedition.org/kernos/566

DOI : $10.4000 /$ kernos.566

ISSN : 2034-7871

Éditeur

Centre international d'étude de la religion grecque antique

Édition imprimée

Date de publication : 1 janvier 1993

Pagination : 381-384

ISSN : 0776-3824

\section{Référence électronique}

Vinciane Pirenne-Delforge, "A. Avagianou, Sacred Marriage in the Rituals of Greek Religion », Kernos

[En ligne], 6| 1993, mis en ligne le 07 avril 2011, consulté le 24 septembre 2020. URL : http:// journals.openedition.org/kernos/566 ; DOI : https://doi.org/10.4000/kernos.566 
L'A. insiste avec raison sur l'importance de l'épopée homérique qui a fortement humanisé et personnalisé les dieux. L'œuvre d'Homère a marqué profondément le reste de la littérature grecque et le poète exprime, en cette matière comme en d'autres, des positions originales qui ne correspondent pas à ce que ferait attendre son époque.

En reconnaissant les différences essentielles qui existent entre nos conceptions religieuses et celles des Grecs, l'A. souligne enfin le souci de la religion grecque de «maintenir, à travers cette communication même [la prière], une distance que rien ne doit compromettre", et le rôle

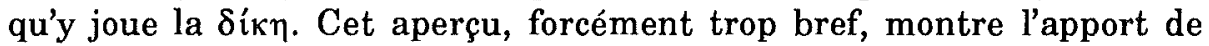
l'ouvrage qu'aucun spécialiste de la religion grecque ne pourra ignorer.

Paul WATHELET (Liège)

\section{Aphrodite AVAGLANOU, Sacred Marriage in the Rituals of Greek Religion, Bern, Peter Lang, 1991, 1 vol. 15,3 x 22,5 cm, XV+260 p., 9 dessins (European University Studies. Series 15. Classics, 54). ISBN : 3-261-04432-2.}

Cette dissertation doctorale, dirigée par W. Burkert, se propose de

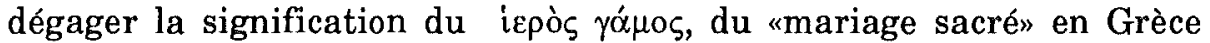
ancienne. En effet, depuis Mannhardt, le sens de cette expression aurait été beaucoup trop étendu, avec Frazer et Klinz notamment, allant jusqu'à désigner tout type d'union en relation avec un contexte magique de fertilité et dont un des partenaires est de nature divine. Pour l'A., le caractère sacré du yó $\mu_{0}$ implique que les deux partenaires doivent être divins - ce qui exclut toute une série de vagabondages divins en territoire humain. De plus, la notion de $\gamma \alpha \dot{\alpha} \mu о \varsigma$ en tant que mariage institutionnalisé et durable exclut les relations fondées sur un rapt. Il s'agit donc de chercher à quels cas précis ces deux principes peuvent être appliqués et quelles différences existent entre le "mariage sacré» et d'autres types de $\mu i \xi_{\varepsilon} \iota \zeta$. Enfin, contre l'interprétation par la «magie sympathique", l'A. entend souligner le caractère initiatique de ce type de mariage.

Puisqu'il s'agit d'étudier un $\gamma \alpha \dot{\alpha} \mu \rho \varsigma$, l'introduction tente, dans une première partie, de reconstituer le plus complètement possible la cérémonie du mariage qui, pour les humains, est une affaire légale, sociale, mais dont le contenu religieux est loin d'être négligeable. Sur base de sources littéraires et de la peinture de vases, la présentation

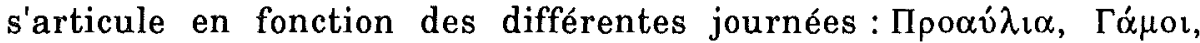

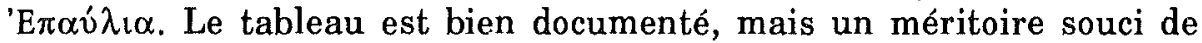
clarté s'affirme parfois au mépris de la complexité des problèmes : les sacrifices préliminaires ne sont pas une rétribution pour la perte de la chasteté (p. 4) (le mariage se doit d'être chaste !), mais bien celle de la virginité; il n'est pas sûr que le loutrophore placé sur la tombe des 
célibataires signifie qu'ils ont atteint dans la mort ce qu'ils n'ont pas vécu dans la vie (p. 6); manger un coing et brûler l'essieu du chariot qui a transporté les jeunes mariés sont des rites que l'on ne peut pas simplement expliquer par l'établissement de la fiancée dans sa nouvelle position (p. 13); le témoignage de Pollux affirmant qu'après le mariage, chaque époux dort une nuit chez ses beaux-parents respectifs, lui avec une petite fille, elle avec un petit garçon, mériterait un traitement plus substantiel qu'une remarque lapidaire sur la qualité d'intégration du rite (p. 15-16) (si l'on peut éventuellement y souscrire pour la mariée, que dire du marié ?), et ce d'autant que Callimaque fait référence à un rite analogue, mais pré-nuptial; le rite du mariage serait le stade final de l'initiation féminine (p. 18), mais que dire de la maternité ?

La deuxième partie de l'introduction porte sur la terminologie du

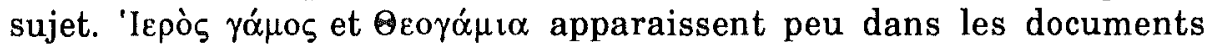
anciens et concernent exclusivement Zeus et Héra (10 fois), et Hadès et Korè (1 fois).

La première partie s'attache à l'étude des mariages sacrés tels que définis dans l'introduction. Le iєpò $\gamma^{\alpha} \alpha$ ó de Zeus et d'Héra (chap. I) serait attesté à Athènes, en Argolide, à Samos, à Platées, à Stymphale, à Cnossos. Pour chaque cas, l'analyse des sources est minutieuse et l'A. fait avec précision le point de la recherche contemporaine. Néanmoins,

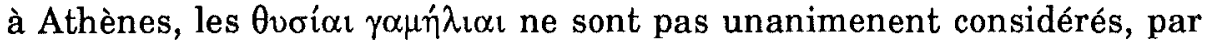
les sources citées p. 28 n. 6, comme les sacrifices matrimoniaux au sein de la phratrie, mais bien, pour certains, comme des sacrifices engagés par les pères pour leur fille; en Argolide, le recouvrement de sa virginité par Héra chaque année dans la fontaine Kanathos n'est pas tant l'indice d'une revitalisation du pouvoir divin accordé à la virginité (pré-hellénique ?) que la condition même d'un rituel périodique; à Platées, si la référence au mariage est patente dans la célébration des Daidala, le foudroiement de la "fiancée» trouve une justification peu satisfaisante dans la référence au mythe de Sémélè quand elle est foudroyée, l'union a déjà été consommée - et dans la fusion, toute hypothétique de Plataia-Héra-Sémélè. Ces quelques réserves ne doivent pas occulter le mérite de ce chapitre qui vise à repenser en ses références humaines la cérémonie du mariage exaltée par des divinités.

Le 2 e chapitre de cette 1re partie propose une liste des représentations présumées du mariage sacré de Zeus et d'Héra, dont les exemples les plus célèbres sont la figuration du couple sur la frise est du Parthénon et sur une des métopes de l'Héraion de Sélinonte, qui reçoivent toutes deux un traitement plus important et très intéressant. Les identifications proposées pour les 46 entrées de ce catalogue sont parfois conjecturales, mais présentées avec les réserves d'usage. Le chapitre III qui les 
interprète compare les représentations des mariages humains et celles de l'hiérogamie, mais les critères discriminants sont peu clairs. La seule conclusion ferme est celle de la solidarité entre les images censées se situer dans la sphère divine et celles qui figurent les humains. Le chapitre IV quitte momentanément Zeus et Héra pour s'attacher au mariage particulier d'Hadès et de Korè. Dérogeant apparemment aux critères fixés d'emblée, l'A. voit dans ce qu'il faut bien appeler un rapt la dramatisation de la "première fois" dans le mariage. Korè est le "prototype" mythique de la fiancée transférée d'un groupe humain à un autre. Il s'agit tout autant de l'expression de valeurs sociales que d'une interprétation de l'expérience de la jeune fille. L'A. rejette donc sans hésitation les analyses qui voyaient dans le rapt de Korè une sorte de métaphore du cycle agricole pour privilégier la dimension humaine du rite. Si cet enlèvement préliminaire est intégré malgré tout dans les "mariages sacrés", c'est parce que la fiancée est donnée par Zeus à Hadès et que son union avec le dieu des enfers est institutionnalisée, notamment au travers des privilèges qu'elle reçoit en tant que souveraine des morts : Korè est devenue Perséphone. On suivra aisément l'A. dans cette voie; mais pourquoi imposer au lecteur l'accumulation des textes anciens évoquant l'enlèvement de Korè et son intégration comme épouse? Il s'agit de montrer combien est pertinent le schéma tripartite de Van Gennep (séparation, transition, incorporation) dans l'analyse du mythe et des rites - ces derniers se limitant au seul exemple le moins mal connu, à savoir une célébration sicilienne. Cet exposé aurait gagné à plus de concision. Quant à la représentation $d u$ motif, c'est sur les plaquettes locriennes que l'on en trouve les exemples les plus intéressants.

La 2e partie du travail concerne les «rapt divins» que l'A. se refuse à qualifier de "mariages sacrés". Il s'agit du viol de Déméter par Poséidon sous une forme chevaline et de l'union de la même Déméter et de Iasion dans une jachère trois fois labourée. Après un long détour par la question des origines possibles du mythe de l'union zoomorphe de Déméter et de Poséidon, l'A. réfute l'explication commune qui voit dans ce viol une manifestation de la fertilité et, fidèle à sa thèse, en privilégie la dimension humaine: Déméter est l'image archétypale de la femme soumise par l'homme, mais, contrairement à la situation d'Héra, ce "sacrifice" n'est pas légitimé par un mariage institutionnalisé. Quant à l'union de Déméter et de Iasion, elle n'entre pas non plus dans la catégorie des mariages sacrés, car, d'après l'Odyssée, Iasion est un mortel puni par Zeus. La comparaison avec l'union d'Aphrodite et d'Anchise, que l'A. évoque en passant, aurait mérité un traitement plus substantiel, car, contrairement à ce que l'A. affirme, Aphrodite ne choisit pas son partenaire, c'est Zeus qui la pousse dans les bras du Troyen. De surcroît, si Aphrodite menace Anchise de mort en cas 
d'indiscrétion de sa part, son foudroiement est une donnée marginale dans la tradition postérieure. Quant à la comparaison, simplement énoncée, avec l'union d'Ishtar et de Gilgamesh, ou encore celle de la Déesse syrienne et du prince, plutôt que de n'en rien dire de plus, l'A. aurait dû en rester à ses intentions préliminaires de ne pas parler de l'Orient (p. XV).

La 3e et dernière partie étudie le cas étrange et isolé de l'union, dans le temps de l'histoire, de Dionysos et de la Basilinna athénienne. L'ambiguité du rite secret est bien mise en évidence : il s'agit de la $\sigma \hat{u} \mu \mu \imath \xi_{\iota \varsigma}$ d'un immortel et d'une mortelle, mais dans un cadre légal. On souscrira volontiers à l'hypothèse qui fait de l'archonte-roi le Dionysos du rituel. Il est en effet difficilement imaginable que le prêtre ait tenu ce rôle, ce qui impliquerait la reconnaissance d'un adultère. Il s'agit d'une tentative réussie pour combler l'écart entre mortalité et immortalité au travers d'un rituel symbolique répété. Les éléments de la légitimation du "mariage" apparaissent dans l'art - un catalogue des représentations présumées de ce rite est proposé.

L'ouvrage se referme sur une brève conclusion qui reprend très clairement les différents acquis du travail. On trouve aussi une liste des abréviations utilisées, une bibliographie imposante - et multilingue - et de brefs index. Deux titres supplémentaires auraient pu figurer dans la bibliographie: Pierre BRULÉ, La fille d'Athènes, Paris, 1987; Giulia SISSA, Le corps virginal, Paris, 1987.

Le "mariage sacré» méritait d'être à nouveau étudié et le travail d'A. Avagianou rend au thème sa juste dimension. La perspective anthropologique prend nettement le pas sur les interprétations naturalistes habituelles et ce contre-pied est sans aucun doute salutaire. Néanmoins, la solidarité entre la fécondité humaine - intimement liée

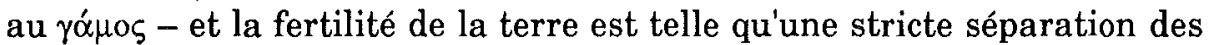
domaines est peut-être illusoire.

Bien documenté, fournissant à chaque étape un état de la question précis, cet ouvrage a cependant, me semble-t-il, conservé à l'excès la forme de la thèse. Il aurait fallu alléger certaines démonstrations, des rapprochements de "religion comparée», trop brefs, auraient dû être évités, de nombreux textes grecs auraient dû n'apparaitre qu'en notes. De même, pourquoi traduire certains extraits et pas les autres? Il reste de nombreuses coquilles, tant dans le cours du texte que dans les références en notes et la bibliographie en langue française, que l'on félicite l'A. d'avoir consultée, n'a guère été soignée dans la forme. Quant aux divers catalogues de sources figurées, il faut être iconologue pour juger de leur qualité. Ne pouvant revendiquer une telle compétence, on s'abstiendra dès lors d'émettre un jugement sur ce point.

Vinciane PIRENNE-DELFORGE (Liège) 